\title{
THE EFFECT OF LOSS-AVERSE BEHAVIOUR ON CAPACITY PORTFOLIO PLANNING FOR POWER SYSTEMS
}

\author{
Bao, X.* \& Jiang, Y. P.** \\ ${ }^{*}$ Zhejiang Institute of Administration, West Wenyi Road 1000, Hangzhou, China \\ ${ }^{* *}$ Department of Industrial \& Systems Engineering, National University of Singapore, \\ 1 Engineering Drive 2, Singapore \\ E-Mail: goldbxing@163.com, yipingjiang@yeah.net $\left({ }^{* *}\right.$ Corresponding author $)$
}

\begin{abstract}
A decision maker seldom behaves completely rationally when deciding on the investment in capacity portfolio for a power system. Thus his decision will certainly show some bias when compared with a truly risk-neutral one. In order to provide an in-depth analysis of such bias, this paper studies the issues influencing capacity portfolio planning for a power system when the decision maker is lossaverse. The investment decision is the result of the decision maker aiming to maximize utility, and the effect of this is investigated using a modified newsvendor model, in which the probability of abnormal electricity demand is considered. Theoretical proofs and numerical simulations are also presented to provide explanations of some counterintuitive findings. We find that bias in the capacity portfolio occurs whenever underage losses are greater than overage losses. Even where significant flexibility is installed, the loss-averse decision maker values dedicated capacity to a greater extent when building redundancy into the system capacity, and all of his decision bias will go towards building-in dedicated capacity rather than flexibility. The most interesting finding is that the probability and variance of abnormal demand are likely to be overestimated, and this will result in investing more dedicated capacity, not only for providing higher service levels in abnormal situations, but also for delivering greater operational profit.

(Received in July 2014, accepted in January 2015. This paper was with the authors 2 months for 1 revision.)
\end{abstract}

Key Words: Capacity Portfolio, Loss-Averse, Uncertain Demand, Newsvendor Model

\section{INTRODUCTION}

As power systems are some of the most important public utilities, the design and investment in their operational capacity are always considered at a strategic level. This is known as capacity portfolio planning. "Being prepared for all eventualities" is a widely acknowledged dictum for mitigating risks caused by any shortfall between capacity and the actual demand. Thus flexible capacity is often installed to provide redundant capacity ready for abnormal situations [1]. However, it is not easy to decide how much flexibility is needed in a capacity portfolio. On one hand, estimation of the statistical parameters is difficult when considering the fluctuating demand for electricity in abnormal situations. On the other hand, decision bias may occur if the decision maker is not completely rational at the investment stage.

Motivated by the research of [2], the issue of capacity portfolio planning by a loss-averse decision maker is investigated in this paper in the context of its effects on investing appropriate capacity for power systems. In October 2012, we conducted a survey at a provincial branch of the State Grid Corporation of China (SGCC), the country's largest electricity provider, with the aim of investigating whether its decision makers were lossaverse when deciding on the capacity portfolio. After in-depth interviews with 3 senior managers and 15 senior engineers, we found that these senior decision makers were noticeably consistent in their loss aversion. All of them strongly agreed that significant redundancy should be built in for minimising the loss in any situation. A certain amount of flexible capacity was built into the capacity portfolio as redundancy, but with an extremely 
low likelihood it being used even in the event of an abnormal situation. Therefore, this begs the question as to whether it results in overinvestment in dedicated capacity?

Two questions have motivated this research. The first is whether a loss-averse decision maker might cause bias in capacity portfolio planning. The second is whether such bias results in overinvestment in dedicated rather than flexible capacity. In this paper, we study on the capacity portfolio planning in the context of the effects that a loss-averse decision maker will have on investment in a power system. The rest of the paper is organized as follows. Following this introduction, we review the literature on capacity portfolio planning in section 2. In section 3, the decisions involved in designing the capacity portfolio for a power system are described. In section 4, a modified newsvendor model is presented to represent the utility function of a loss-averse decision maker, and theoretical proofs are presented. In section 5, we provide numerical simulations of some critical parameters. Finally, conclusions and proposals for future research are given in section 6 .

\section{LITERATURE REVIEW}

Capacity portfolio planning refers to determination of the volumes, types and scheduling of installation of an operational system, and studies of this are widely presented in the related literature [3-5]. Such strategic planning plays an important role in the development of power systems. Once the investment has been made, any subsequent modifications to the capacity portfolio would result in an additional multi-million dollar financial burden, and require several years for installing the new capacity. A properly designed and financed capacity portfolio is not only beneficial in helping the company to acquire market competitiveness and in enhancing operational efficiency; it also provides the ability to hedge risk across a range of uncertainties [6]. The works most related to our research are those on decision-making with respect to building flexibility into the capacity portfolio in order to hedge against such uncertainties [7].

The first stream in flexibility research is the more technical: that of determining the optimal or desired capacity portfolio. Stochastic capacity planning models have been developed to investigate the extent of investment in capacity required for dealing with uncertainties of demand [8]. The mean and variance of uncertain demand under different scenarios have been carefully examined ahead of presenting heuristic algorithms or simulations [9, 10]. Most of these mathematical programming models are aimed at minimizing the gap between capacity and actual demand, while maximizing the operational profits. In some research [11], further uncertainties beyond those of fluctuating demand, and of price and yield, have been included to add greater reality to the above models. Other researchers have considered the expansion of flexible capacity, while the use of different kinds of flexibility and the reconfiguration of dedicated capacity have been studied using stochastic dynamic programming approaches $[12,13]$. Besides decisions with respect to the volumes and types of capacity, the timings of investment in flexibility, as part of a scenario of capacity expansion, have also been considered in some studies $[14,15]$.

The second stream of research concerns the value of flexibility in mitigating risks, by empirical and analytical models. Through CVaR-based newsvendor models, flexibility has been theoretically proved to be beneficial for operational profit under unreliable supply networks, when risk-averse decision makers are assumed [16, 17]. Some general utility functions and mean-variance formulations are introduced to the investment of flexibility by a risk-averse newsvendor, and the conclusion of investing more flexibility roughly proportionally to demand variance is drawn by [7]. The value of flexibility is further highlighted by the empirical study presented in [18], where the intrinsic flexibility built in by using multifunctional capacity provides more advantages in terms of supply availability 
during any disruptions when compared with the use of external flexibility through coordination of the supply chain. Further benefits of flexibility have been identified by other researchers who investigated the long-run stock price performances and equity risks of 827 samples from 1989 to 2000 [19]. However, there are some different opinions on the value of investment in flexibility. There is only limited benefit to operational profits, even though the cost of investment in the technology to provide flexibility is zero [20].

However, there are apparent differences in the decisions made by risk-averse and lossaverse decision makers. To mitigate risk, the former pay greater attention to the extending variance of uncertainty, which reflects the risk exposure [7]. While the latter, tries to avoid any loss caused by mismatch gap between the capacity and actual demand [21]. Some of the optimal decisions given by loss-averse models may never actually occur in the riskaverse/risk-neutral models [22]. More evidence on the different decision paths of these two types of decision maker can be seen in researches of financial, economic, marketing and organizational behaviour [23]. However, little research could be found in the field of operational management. To the best of our knowledge, we are the first to investigate lossaversion behaviour in respect of the problem of capacity portfolio planning for power systems.

\section{PROBLEM DESCRIPTION AND NOTATIONS}

In this research, we consider that the decision maker invests in two kinds of equipment in respect of electricity demand fluctuating in normal and abnormal situations. The first kind of equipment is the existing generation equipment, which operates more efficiently and has lower installation and operational costs, while its disadvantage is obviously in its inability to provide capacity expansion to meet other situations. The second is advanced equipment which can expand its capacity to meet sharp upwardly shifting electricity demand; however its installation and operational costs are much greater than those of the former. In this paper, we refer to the advanced and existing generation equipment as flexible and dedicated capacity respectively. Therefore, here, we have defined 'capacity portfolio' simply as the total volumes and proportions of these two kinds of capacity.

There is an obvious difference in the considerations taken into account by a risk-averse or a loss-averse decision maker in weighting the profit and loss. A risk-averse decision maker might decide on the capacity portfolio only by consideration of the increasing variance of an uncertain electricity demand while its mean remains constant [7]. Thus, the aim of a riskaverse decision maker is to try to reduce the exposure to risk. However, a loss-averse decision maker will aim to minimize any financial losses, and would therefore consider both the mean and the variance, as well as the probability of an abnormal electricity demand. The design of a capacity portfolio by a loss-averse decision maker is illustrated in Fig. 1. In this paper, both normal and abnormal electricity should be satisfied by flexible and dedicated capacity in different capacity expansion scenario. The maximum output of the capacity portfolio is designed to provide sufficient electricity in both normal and abnormal situations. We shall consider only an upward-shifting of the mean of the abnormal demand, because the demands resulting from a downward-shift in the mean would, of course, be satisfied by the capacity portfolio.

The problem of a loss-averse decision maker deciding the capacity portfolio will be presented in newsvendor models. The following notations are used in the models.

\section{Demand related parameters}

$x$ and $y$ are random electricity demands in normal and abnormal situations respectively. Both of them have a Gaussian distribution. $f(x), g(y), F(x)$ and $G(y)$ are the density and distribution functions respectively. 


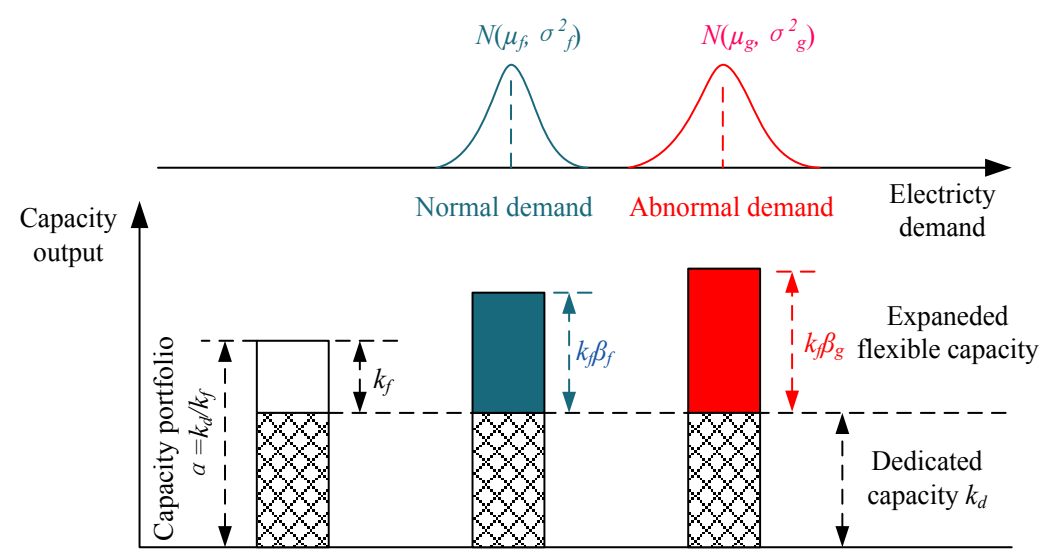

Figure 1: Flexible capacity expansion when electricity demand fluctuates normally and abnormally.

$\left(\mu_{f}, \sigma_{f}^{2}\right)$ and $\left(\mu_{g}, \sigma_{g}^{2}\right)$ are the means and variances of the normal and abnormal electricity demand. $\mu_{g}>\mu_{f}$, but, according to historical data, $\mu_{g}$ has seldom exceeded $\mu_{f}$ by more than $25 \%$.

Capacity related parameters

$k_{d}$ and $k_{f}$ are the volume of the dedicated and flexible capacity respectively. Thus $\left[k_{d}, k_{f}\right]$ is the capacity portfolio.

$\alpha=k_{d} / k_{f}$ and is the capacity structure ratio. For convenience in constructing the mathematical model without losing any general applicability, we set $k_{f}=1$ in this paper, thus $\alpha=k_{d}$.

$\beta_{f}$ and $\beta_{g}$ are the expansion coefficients of the flexible capacity in normal and abnormal situations. Noted that $\left(\beta_{g}-\beta_{f}\right) \geq\left(\mu_{g}-\mu_{f}\right)$, or the demand in the abnormal situation cannot be satisfied. And $\beta_{g}>\beta_{f} \geq 1$.

$\left(k_{d}+\beta_{f} k_{f}\right)=\left(\alpha+\beta_{f}\right)$ and $\left(k_{d}+\beta_{g} k_{f}\right)=\left(\alpha+\beta_{g}\right)$ are the respective outputs of the capacity portfolio when electricity demand fluctuates normally and abnormally. $\left(\alpha+\beta_{g}\right)$ is also the maximum output of the capacity portfolio.

\section{Cost related parameters}

$c$ is the operational cost of dedicated capacity per unit.

$q(\beta)$ is the operational cost function of flexible capacity per unit at the expansion coefficient of $\beta$, and its marginal cost is $q^{\prime}(\beta)>c$. According to the engineers interviewed, $q^{\prime}(\beta)$ is 3 to 4 times greater than $c$.

$r$ is the revenue of per electricity demand that is satisfied.

$w$ is the penalty of per electricity demand that is unsatisfied. It can be either the punishment from electricity consumer or regulator. $w>q^{\prime}(\beta)$, or else no flexible capacity will be expanded in abnormal situation.

$s$ is the salvage cost of excessive capacity per unit of the power system, such as the overproduced electricity could be stored by pumped-storage hydro-electric power station.

And $w>r>c>s$.

Profit and utility related parameters

$\pi$ is the operational profit for the given capacity portfolio.

$\lambda$ is the loss aversion coefficient of the decision maker, where $\lambda \geq 1$. The higher the value of $\lambda$, the more loss-averse the decision maker. If the decision maker is risk-neutral $\lambda=1$.

$U(\pi)$ is the utility function of a loss-averse decision maker according to perspective theory[2]. This is a piecewise utility function, $U(\pi)=\pi$ when $\pi \geq 0$, and $U(\pi)=\lambda \pi$ when 
$\pi<0$. This implies that the decision maker is more sensitive to losses than to profits of equivalent size.

\section{Probability related parameter}

$p$ is the probability of there being abnormal electricity demand, while $(1-p)$ is the probability of normal electricity demand.

\section{MATHEMATICAL MODEL}

It is the objective of a decision maker to maximize his utility function when deciding the optimal capacity portfolio. However, the utility functions of risk-neutral and loss-averse decisions are rather different. A risk-neutral decision maker will aim to maximise the total expected profit, with profit and loss of the same amount being treated no differently in his utility function. However, for the utility function of a loss-averse decision maker, loss is weighted more heavily than the same amount of profit, thus there will be obvious decision bias in his optimal capacity portfolio when compared with a risk-neutral one.

Before constructing the utility function of a loss-averse decision maker, we would like to present the risk-neutral decision maker's utility function in eq. (1), which is an expected profit function weighted by the probability of abnormal electricity demand according to the Von Neumann and Morgenstern's Rule.

$$
E[\pi(\alpha, x, y)]=(1-p) E\left[\pi_{f}(\alpha, x)\right]+p E\left[\pi_{g}(\alpha, y)\right]
$$

$\pi_{f}(\alpha, x)$ and $\pi_{g}(\alpha, y)$ are the respective profit functions when demand fluctuates normally and abnormally, and are derived as follows:

$$
\begin{aligned}
\pi_{f}(\alpha, x) & =\operatorname{rmin}\left[x,\left(\alpha+\beta_{f}\right)\right]+s\left[\left(\alpha+\beta_{f}\right)-x\right]^{+}-w\left[x-\left(\alpha+\beta_{f}\right)\right]^{+}-\left[c \alpha+q\left(\beta_{f}\right)\right] \\
\pi_{g}(\alpha, y) & =\operatorname{rmin}\left[y,\left(\alpha+\beta_{g}\right)\right]+s\left[\left(\alpha+\beta_{g}\right)-y\right]^{+}-w\left[y-\left(\alpha+\beta_{g}\right)\right]^{+}-\left[c \alpha+q\left(\beta_{g}\right)\right]
\end{aligned}
$$

The first item in each of eqs. (2) or (3) is the revenue from electricity demand satisfied by the output of the capacity portfolio. The second is the salvage cost when overproduced electricity reclaimed by pumped-storage hydro-electric power station. The third is the penalty cost when not enough electricity is produced. And the fourth item is the operational costs of dedicated and flexible capacity.

$E[\pi(\alpha, x, y)]$ is obviously a newsvendor model, and the optimal capacity structure ratio $\alpha^{*}$ will be ensured by maximizing the expected profits. Therefore we can present the following proposition:

Proposition 1. In the context of a risk-neutral decision maker, $E[\pi(\alpha, x, y)]$ is strictly concave, there is a unique value of $\alpha^{*}$ that makes $E\left[\pi\left(\alpha^{*}, x, y\right)\right]=\max \{E[\pi(\alpha, x, y)]\}$, and $\alpha^{*}$ satisfies the eq. (4)

$$
(1-p) F\left(\alpha^{*}+\beta_{f}\right)+p G\left(\alpha^{*}+\beta_{g}\right)=\frac{r+w-c}{r+w-s}
$$

$F\left(\alpha^{*}+\beta_{f}\right)$ and $G\left(\alpha^{*}+\beta_{g}\right)$ are the utility function of the power system under normal and abnormal demand respectively.

The proof of proposition 1 is rather simple, thus we have omitted here. Eq. (4) could be obtained by solving $\frac{\partial \mathrm{E}[\pi]}{\partial \alpha}=0$.

According to [24], loss will be found whenever there is a mismatch between the planned capacity and uncertain electricity demand. Two kinds of loss can be defined. An overage loss results from the power system producing more electricity than is necessary, while underage losses are the result of insufficient electricity provision compared to the actual demand. Both of overage and underage losses are weighted more than revenue. Therefore, in order to construct the utility function of a loss-averse decision maker, overage and underage losses 
should be defined in terms of the actual electricity demand $x_{i}$ or $y_{i}$ that make $\pi_{f}\left(\alpha, x_{i}\right)$ or $\pi_{g}\left(\alpha, y_{i}\right)$ equal to zero.

Lemma. There are $x_{1}$ and $x_{2}$, or $y_{1}$ and $y_{2}$ that make $\pi_{f}(\alpha, x)=0$ or $\pi_{g}(\alpha, y)=0$. And $x_{1}=\left\{[c-s] \alpha+\left[q\left(\beta_{f}\right)-s \beta_{f}\right]\right\} /(r-s), x_{2}=\left\{[r+w-c] \alpha+\left[(r+w) \beta_{f}-q\left(\beta_{f}\right)\right]\right\} / w$, $y_{1}=\left\{[c-s] \alpha+\left[q\left(\beta_{g}\right)-s \beta_{g}\right]\right\} /(r-s), y_{2}=\left\{[r+w-c] \alpha+\left[(r+w) \beta_{g}-q\left(\beta_{g}\right)\right]\right\} / w$. $x_{1} \leq\left(\alpha+\beta_{f}\right) \leq x_{2}$ and $y_{1} \leq\left(\alpha+\beta_{g}\right) \leq y_{2}$. Overage loss will be caused when $x<x_{1}$ or $y<y_{1}$, while underage loss will be caused when $x>x_{2}$ or $y>y_{2}$.

As this lemma can be easily proved when eqs. (2) and (3) equal zero, the proof has been omitted.

Eqs. (5) and (6) are the utility functions of a loss-averse decision maker. Both the overage and underage losses are weighted by a loss-aversion coefficient $\lambda$.

$$
\begin{aligned}
U_{f}\left[\pi_{f}(\alpha, x)\right] & =E\left[\pi_{f}(\alpha, x)\right]+(\lambda-1)\left\{E\left[\pi_{f}\left(\alpha, x \leq x_{1}\right)\right]+E\left[\pi_{f}\left(\alpha, x \geq x_{2}\right)\right]\right\} \\
U_{g}\left[\pi_{g}(\alpha, y)\right] & =E\left[\pi_{g}(\alpha, y)\right]+(\lambda-1)\left\{E\left[\pi_{g}\left(\alpha, y \leq y_{1}\right)\right]+E\left[\pi_{g}\left(\alpha, y \geq y_{2}\right)\right]\right\}
\end{aligned}
$$

Therefore, by substituting $\pi_{f}$ and $\pi_{g}$ with $U_{f}$ and $U_{g}$, we can obtain the expected utility function of a loss-averse decision maker as $U[\pi(\alpha, x, y)]$ as in eq. (7). The optimal capacity portfolio $\alpha^{* *}$ can be obtained by maximizing $U[\pi(\alpha, x, y)]$, which is also a variant of the newsvendor.

$$
U[\pi(\alpha, x, y)]=(1-p) U_{f}\left[\pi_{f}(\alpha, x)\right]+p U_{g}\left[\pi_{g}(\alpha, y)\right]
$$

Proposition 2. $U[\pi(\alpha, x, y)]$ is strictly concave with $\alpha$, and there is an unique $\alpha^{* *}$ that makes $U\left[\pi\left(\alpha^{* *}, x, y\right)\right]=\max \{U[\pi(\alpha, x, y)]\}$, and where $\alpha^{* *}$ satisfies eq. (8):

$$
\begin{gathered}
(1-p)\left\{\left[A \bar{F}\left(\alpha^{* *}+\beta_{f}\right)-B F\left(\alpha^{* *}+\beta_{f}\right)\right]+(\lambda-1)\left[A \bar{F}\left(x_{2}\right)-B F\left(x_{1}\right)\right]\right\}+ \\
p\left\{\left[A \bar{G}\left(\alpha^{* *}+\beta_{g}\right)-B G\left(\alpha^{* *}+\beta_{g}\right)\right]+(\lambda-1)\left[A \bar{G}\left(y_{2}\right)-B G\left(y_{1}\right)\right]\right\}=0
\end{gathered}
$$

where $\bar{F}(\cdot)=1-F(\cdot), \bar{G}(\cdot)=1-G(\cdot), A=r+w-c$ and $B=c-s$.

As the proof of proposition 2 is similar to that for proposition 1, we have omitted it here.

Since $\bar{F}(\cdot)$ and $\bar{G}(\cdot)$ are the probabilities that the capacity portfolio $\alpha^{* *}$ can not satisfied in the case of uncertain electricity demand, $A \bar{F}(\cdot)$ and $A \bar{G}(\cdot)$ are the underage losses, while $B F(\cdot)$ and $B G(\cdot)$ are the overage losses.

Corollary 1. The more loss-averse the decision maker is, the greater amount of flexible capacity will be invested. That is $\frac{\partial \alpha^{* *}}{\partial \lambda}<0$.

As the proof of corollary 1 is very simple, it has been omitted.

Corollary 2. When variance of demand increases in abnormal situation, more dedicated capacity will be invested when $p$ increases, and vice versa. Specifically, $\frac{\partial \alpha^{* *}}{\partial p}>0$, when $\frac{\sigma_{g}}{\sigma_{f}}>1$, and $\frac{\partial \alpha^{* *}}{\partial p}<0$ when $\frac{\sigma_{g}}{\sigma_{f}}<\frac{\mu_{g}-\beta_{g}}{\mu_{f}-\beta_{f}}<1$.

Proof of corollary 2. We can obtain $\frac{\partial \alpha^{* *}}{\partial p}=\frac{(A+B)\left[F\left(\alpha^{*}+\beta_{f}\right)-G\left(\alpha^{*}+\beta_{g}\right)\right]}{(1-p) f\left(\alpha^{*}+\beta_{f}\right)+p g\left(\alpha^{*}+\beta_{g}\right)}$ from eq. (8). It is evident that the sign of $\frac{\partial \alpha^{* *}}{\partial p}$ depends on the sign of $\left[F\left(\alpha^{* *}+\beta_{f}\right)-G\left(\alpha^{* *}+\beta_{g}\right)\right]$, which is equivalent to $\left[\frac{\alpha^{* *}+\beta_{f}-\mu_{f}}{\sigma_{f}}-\frac{\alpha^{* *}+\beta_{g}-\mu_{g}}{\sigma_{g}}\right]$ according to the normal approximation approach. $\left[\frac{\alpha^{* *}+\beta_{f}-\mu_{f}}{\sigma_{f}}-\frac{\alpha^{* *}+\beta_{g}-\mu_{g}}{\sigma_{g}}\right]=\frac{1}{\sigma_{f} \sigma_{g}}\left[\left(\sigma_{g}-\sigma_{f}\right) \alpha^{* *}+\sigma_{f}\left(\mu_{f}-\beta_{f}\right)\left(\frac{\sigma_{g}}{\sigma_{f}}-\frac{\mu_{g}-\beta_{g}}{\mu_{f}-\beta_{f}}\right)\right]$. Because $\frac{\mu_{g}-\beta_{g}}{\mu_{f}-\beta_{f}}<1$ according to section $3, \frac{\partial \alpha^{* *}}{\partial p}>0$ when $\frac{\sigma_{g}}{\sigma_{f}}>1$ and $\frac{\partial \alpha^{* *}}{\partial p}<0$ when $\frac{\sigma_{g}}{\sigma_{f}}<\frac{\mu_{g}-\beta_{g}}{\mu_{f}-\beta_{f}}<1$.

Corollary 3. More dedicated capacity will be invested if the penalty increases, that is $\frac{\partial \alpha^{* *}}{\partial w}>0$ 
As the proof of corollary 3 is very simple it has been omitted. Similarly, we can obtain $\frac{\partial \alpha^{* *}}{\partial r}>0, \frac{\partial \alpha^{* *}}{\partial s}>0$ and $\frac{\partial \alpha^{* *}}{\partial c}<0$.

Corollary 4. The bias of $\left(\alpha^{* *}-\alpha^{*}\right)$ depends on the difference between the underage and overage losses. (1) $\alpha^{* *}-\alpha^{*}>0$ when $A \bar{F}\left(x_{2}\right)>B F\left(x_{1}\right)$ and $A \bar{G}\left(y_{2}\right)>B G\left(y_{1}\right)$. (2) $\alpha^{* *}-\alpha^{*}<0$ when $A \bar{F}\left(x_{2}\right)<B F\left(x_{1}\right)$ and $A \bar{G}\left(y_{2}\right)<B G\left(y_{1}\right)$. (3) $\alpha^{* *}=\alpha^{*}$ when $A \bar{F}\left(x_{2}\right)=B F\left(x_{1}\right)$ and $A \bar{G}\left(y_{2}\right)=B G\left(y_{1}\right)$.

Proof of corollary 4. It can be obtained that $\frac{\partial U}{\partial \alpha}=(\lambda-1)\left\{(1-p)\left[A \bar{F}\left(x_{2}\right)-B F\left(x_{1}\right)\right]+\right.$ $\left.p\left[A \bar{G}\left(y_{2}\right)-B G\left(y_{1}\right)\right]\right\}+\frac{\partial E[\pi]}{\partial \alpha}$ according to eq. (7). In the first case, suppose that $\alpha^{* *}>\alpha^{*}$, and $\frac{\partial E\left[\pi\left(\alpha^{* *}\right)\right]}{\partial \alpha}<\frac{\partial E\left[\pi\left(\alpha^{*}\right)\right]}{\partial \alpha}=0$ because $\frac{\partial^{2} E[\pi(\alpha)]}{\partial \alpha^{2}}<0$ according to proposition 1, only $A \bar{F}\left(x_{2}\right)>B F\left(x_{1}\right)$ and $A \bar{G}\left(y_{2}\right)>B G\left(y_{1}\right)$ could make $\frac{\partial U\left(\alpha^{* *}\right)}{\partial \alpha}=0$. We can also obtain similar outcomes for the other two cases.

If the bias $\left(\alpha^{* *}-\alpha^{*}\right)$ is defined as the over-redundancy of the capacity portfolio of the loss-averse decision maker, it is interesting that $\left(\alpha^{* *}-\alpha^{*}\right)$ will result only from dedicated capacity rather than from flexible capacity.

\section{NUMERICAL SIMULATIONS}

In this section, numerical simulation will be carried out to investigate the decision-making behaviour of a loss-averse decision maker. In sections 5.1 and 5.2, numerical analysis of the variance, probability and the effects of penalties are presented to investigate their influence on the capacity portfolio decisions and the extent of the resulting bias. In section 5.3, the operational profits are calculated to verify the conclusions given by the aforementioned simulations. The basic parameters in this section are taken as $\mu_{f}=10, \mu_{g}=12.5, \sigma_{f}=0.5$, $\beta_{f}=1, \beta_{g}=4, r=100, c=80, s=50$ and $q(\beta)=3 c \beta . \sigma_{g}$, while $\lambda$ and $w$ will be the changing parameters.

\subsection{Sensitivity analysis of variance}

The variance of abnormal electricity demand is particularly important when deciding on the capacity portfolio, according to [25]. Under the basic parameter settings, with $\lambda=2, w=200$ and $p=0 \sim 0.5$, a loss-averse decision maker would invest in dedicated capacity as in Fig. 2. A more widely fluctuating abnormal demand is indicated when $\sigma_{g} / \sigma_{f}>1$, while the opposite is the case when $\sigma_{g} / \sigma_{f} \leq 1$.

According to Fig. 2, it is quite counter-intuitive that a loss-averse decision maker would tend to invest more dedicated capacity when $\sigma_{g}$ increases. This finding is similar to that of [20], and investment in flexible capacity is made merely for profit maximization under normal operational conditions rather than for coping with abnormal electricity demand. A high capacity portfolio volume with more dedicated capacity will contribute an increase in the service level of the power system able to meet the demands of both normal and most abnormal situations.

According to the interviews which we conducted, almost every decision maker within the SGCC would normally make decisions which result in the capacity portfolio falling within the grey-coloured area in Fig. 2. As a consequence, there will be an emphasis on more dedicated capacity being invested, resulting in built-in redundancy. This provides the answer to the question proposed in our introduction. 


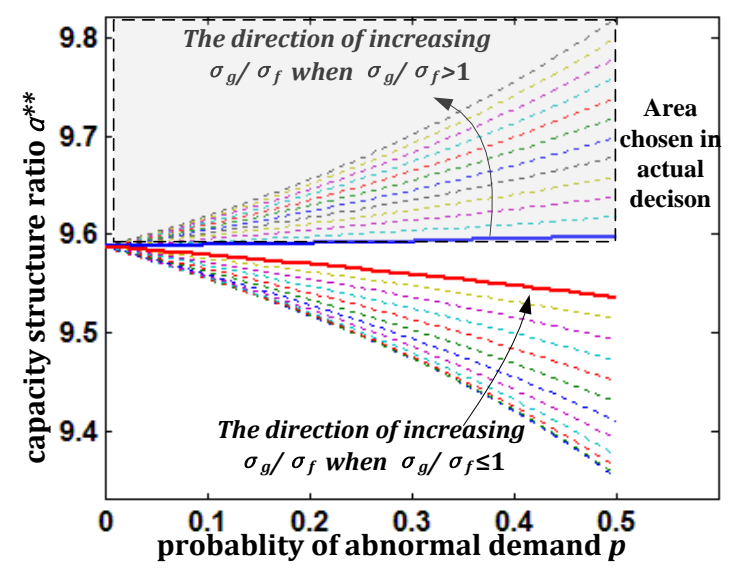

Figure 2: $\alpha^{* *}$ when $\sigma_{g} / \sigma_{f}=[1.05,6.55]$ and $[0.78,0.9]$ with increments of 0.5 and 0.01 respectively.

\subsection{Capacity portfolio bias simulations}

Under the basic parameter settings, the underage loss is greater than the overage loss, thus the bias of $\left(\alpha^{* *}-\alpha^{*}\right)$ is positive. Since almost every decision maker is inclined to provide a capacity portfolio decision resulting in $\sigma_{g}>\sigma_{f}$, we shall next investigate further in respect of the parameters that impact on this bias.

The first parameter to be investigated in this section is the probability of an abnormal situation occurring, which is illustrated in Table I. What interests us most is the sharp-fall in $\left(\alpha^{* *}-\alpha^{*}\right)$ when $p$ falls below 0.1 , and where there is little difference between $\alpha^{*}$ and $\alpha^{* *}$ with an extremely low $p$, for example if $p=0.0001$. When $p=0$, a loss-averse decision maker behaves in the same way as a risk-neutral one, meaning that no additional redundant capacity would be invested. According to our interviews, the SGCC decision makers would never consider an investment in redundant capacity to cover adverse situations which have an extremely low probability, such as earthquakes and terrorist attacks. This matches the findings reported in [26]. However, the probability of some other abnormal adverse events, such as fire, extremely hot or cold weather, would be overestimated. Therefore, according to Table I, the bias of $\left(\alpha^{* *}-\alpha^{*}\right)$ will increase when $p$ increases. It is actually quite counterintuitive that a loss-averse decision maker would reduce the proportion of flexible capacity in the capacity portfolio, while investing in more dedicated capacity.

Table I: Capacity portfolio bias of $\left(\alpha^{* *}-\alpha^{*}\right)$ when $\lambda=2$ and $w=200$.

\begin{tabular}{|c|c|c|c|c|c|c|c|}
\hline$\alpha^{* *}-\alpha^{*}$ & $p=0.0001$ & $p=0.01$ & $p=0.1$ & $p=0.2$ & $p=0.3$ & $p=0.4$ & $p=0.5$ \\
\hline$\sigma_{g} / \sigma_{f}=3.0$ & $9.8703 \mathrm{e}-6$ & 0.0020 & 0.019 & 0.047 & 0.052 & 0.078 & 0.119 \\
\hline$\sigma_{g} / \sigma_{f}=2.5$ & $5.0726 \mathrm{e}-6$ & 0.0012 & 0.017 & 0.039 & 0.050 & 0.074 & 0.106 \\
\hline$\sigma_{g} / \sigma_{f}=2.0$ & $2.7227 \mathrm{e}-6$ & 0.0010 & 0.016 & 0.032 & 0.049 & 0.069 & 0.090 \\
\hline$\sigma_{g} / \sigma_{f}=1.5$ & $2.6063 \mathrm{e}-6$ & 0.0010 & 0.016 & 0.032 & 0.048 & 0.065 & 0.088 \\
\hline$\sigma_{g} / \sigma_{f}=1.0$ & $1.8321 \mathrm{e}-6$ & 0.0010 & 0.016 & 0.031 & 0.047 & 0.062 & 0.085 \\
\hline
\end{tabular}

The second parameter to be investigated is the penalty $w$, because, according to proposition 2, it contributes the greatest effect in the case of underage loss. The biases are illustrated in Fig. 3, using the same parameters as in Table I. For clarity, only the plots of the biases when $p=0.1$ are illustrated in Fig. 3 because they are fairly similar for increasing values of $p$. The values of $\left(\alpha^{* *}-\alpha^{*}\right)$ in Fig. 3 accord very well with corollary 4 . Taking into consideration corollary 3 , all of the bias will be built up through the installation of dedicated capacity, indicating that dedicated capacity is more highly valued than flexible capacity. 


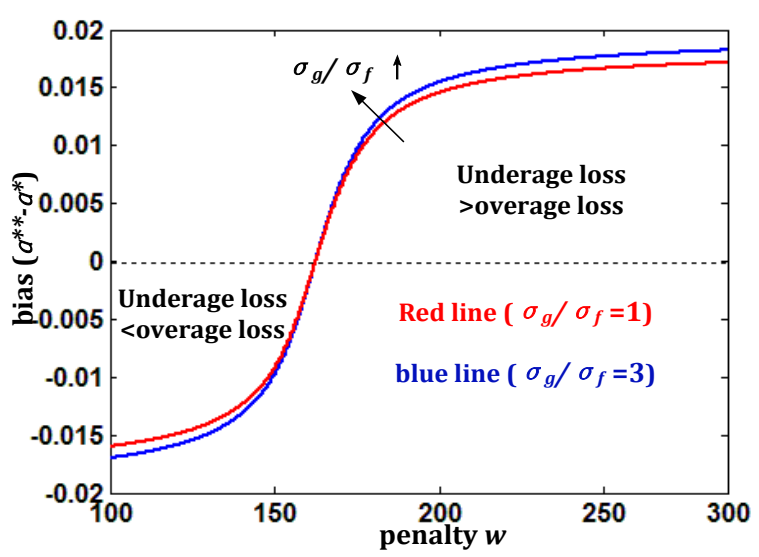

Figure 3: $\left(\alpha^{* *}-\alpha^{*}\right)$ when $w$ varies from 100 to $300, \sigma_{g} / \sigma_{f}=0.9, \sigma_{g} / \sigma_{f}=2, p=0.1$.

\subsection{Operational profit simulations}

According to proposition 1, maximization of operational profit is ensured at $\alpha^{*}$. Therefore, this will fall whenever there is any bias away from $\alpha^{*}$. The size of the operational profits, calculated for the same parameters as used in Table I are illustrated in Fig. 4. The solid and dashed curves in Fig. 4 are the respective operational profits when the decision maker is either risk-neutral or loss-averse.

It is interesting that operational profit increases when $\sigma_{g}$ and $p$ increase. This indicates that almost every decision maker would be inclined to overestimate both the probability and the variance of abnormal electricity demand in order to achieve higher operational profit. This might therefore be the reason why the SGCC decision makers always pay excessive attention to the possibility of extreme hot or cold weather. More capacity is therefore invested not only for hedging risks, but also for higher operational profits. This is consistent with the findings in section 5.2: there will be more dedicated capacity invested when $\sigma_{g}$ and $p$ increase.

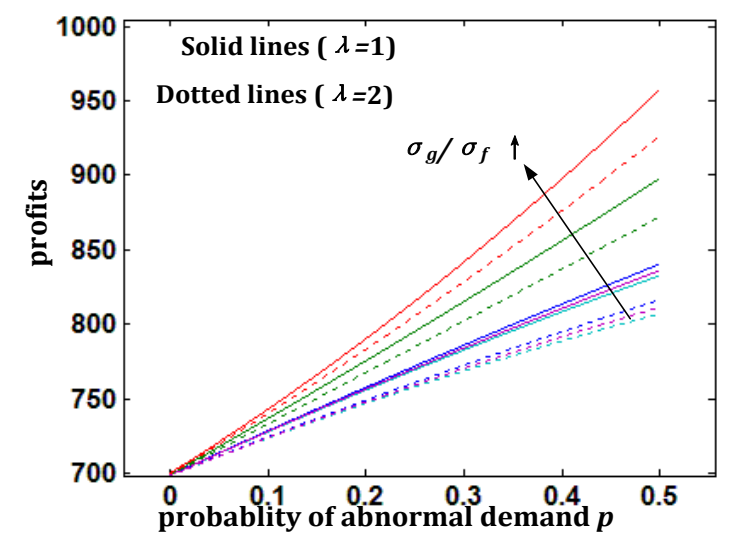

Figure 4: Operational profits when decision makers are loss-averse and risk-natural.

\section{CONCLUSIONS AND FUTURE RESEARCH}

In this paper, we have studied the capacity portfolio planning problem of a power system when the decision maker is loss-averse. A modified newsvendor model has been presented to depict the utility function of such a loss-averse decision maker, and detailed theoretical proofs and numerical simulations have been presented. Several interesting findings have been obtained, and although some of them might be counterintuitive they are helpful in explaining the decisions made by loss-averse decision makers in respect of investment in capacity portfolios. 
Generally, more flexible capacity would be expected to be invested into capacity portfolio with a more loss-averse decision maker. However, the situation might well be reversed if the decision maker overestimates the probability and variance of abnormal electricity demand, as is typically the case in the real-world decision-making. Overinvestment in dedicated capacity is a reasonable decision as it provides higher service levels and operational profits no matter whether the decision maker is loss-averse or risk-neutral. However, a loss-averse decision would tend to over-invest in dedicated capacity, and all the bias in the capacity portfolio will be built by dedicated capacity. Therefore, this is quite similar to the finding of [20], that a loss-averse decision maker will particularly value dedicated capacity when building a higherlevel capacity portfolio.

It is expected that two of the assumptions made in this paper will be relaxed in future modelling studies. The first assumption is that the capacity portfolio problem can be regarded as a single-period decision model. However, the installation of power system capacity is actually made over a long period and in multiple stages. It therefore is appropriate to consider the timing of the capacity portfolio decisions involved. The other assumption is that the use of the loss-aversion coefficient is an accurate representation of a loss-averse decision maker. However the estimation of the loss-aversion coefficient is not easy and is worthy of further study through experimental research [27].

\section{ACKNOWLEDGEMENT}

The authors are grateful for the support of the National Natural Science Foundation of China (Grant No. 7102033), the Research Fund for the Doctoral Program of Higher Education of China (Grant No. 2011332612002), the Zhejiang Provincial Natural Science Foundation of China (Grant No. LQ12G01004), and the Zhejiang Provincial Social Science Foundation of China (Grant No. 13ZJQN048YB).

\section{REFERENCES}

[1] Mitroff, I. I.; Alpaslan, M. C. (2003). Preparing for evil, Harvard Business Review, Vol. 84, No. 4, 109-113

[2] Wang, C. X.; Webster, S. (2009). The loss-averse newsvendor problem, Omega, Vol. 37, No. 1, 93-105, doi:10.1016/j.omega.2006.08.003

[3] Van Mieghem, J. A. (2003). Commissioned paper: Capacity management, investment, and hedging: Review and recent developments, Manufacturing \& Service Operations Management, Vol. 5, No. 4, 269-302, doi:10.1287/msom.5.4.269.24882

[4] Nikonowicz, L.; Milewski, J. (2012). Virtual Power Plants - general review: structure, application and optimization, Journal of Power Technologies, Vol. 92, No. 3, 135-149

[5] Martínez-Costa, C.; Mas-Machuca, M.; Benedito, E.; Corominas, A. (2014). A review of mathematical programming models for strategic capacity planning in manufacturing, International Journal of Production Economics, Vol. 153, 66-85, doi:10.1016/j.ijpe.2014.03.011

[6] Bradley, J. R.; Glynn, P. W. (2002). Managing capacity and inventory jointly in manufacturing systems, Management. Science, Vol. 48, No. 2, 273-288, doi:10.1287/mnsc.48.2.273.254

[7] Van Mieghem, J. A. (2007). Risk mitigation in newsvendor networks: resource diversification, flexibility, sharing, and hedging, Management Science, Vol. 53, No. 8, 1269-1288, doi:10.1287/ mnsc. 1070.0700

[8] Paraskevopoulos, D.; Karakitsos, E.; Rustem, B. (1991). Robust capacity planning under uncertainty, Management Science, Vol. 37, No. 7, 787-800, doi:10.1287/mnsc.37.7.787

[9] Swaminathan, J. M. (2000). Tool capacity planning for semiconductor fabrication facilities under demand uncertainty, European Journal of Operational Research, Vol. 120, No. 3, 545-558, doi: $10.1016 / \mathrm{S} 0377-2217(98) 00389-0$ 
Bao, Jiang: The Effect of Loss-Averse Behaviour on Capacity Portfolio Planning for ...

[10] Huang, K.; Ahmed, S. (2010). A stochastic programming approach for planning horizons of infinite horizon capacity planning problems, European Journal of Operational Research, Vol. 200, No. 1, 74-84, doi:10.1016/j.ejor.2008.12.009

[11] Wu, C.-H.; Chuang, Y.-T. (2010). An innovative approach for strategic capacity portfolio planning under uncertainties, European Journal of Operational Research, Vol. 207, No. 2, 10021013, doi:10.1016/j.ejor.2010.05.015

[12] Chod, J.; Rudi, N. (2005). Resource flexibility with responsive pricing, Operations Research, Vol. 53, No. 3, 532-548, doi:10.1287/opre.1040.0191

[13] Narongwanich, W.; Duenyas, I.; Birge, J. R. Optimal portfolio of reconfigurable and dedicated capacity under uncertainty, from http://users.iems.northwestern.edu/ jrbirge/html/misc_papers/ RMSDMSportfolio.pdf, accessed on 11-10-2014

[14] Meixell, M. J.; Wu, S. D. (2001). Scenario analysis of demand in a technology market using leading indicators, IEEE Transactions on Semiconductor Manufacturing, Vol. 14, No. 1, 65-75, doi: $10.1109 / 66.909656$

[15] Chen, Y.-Y.; Chen, T.-L.; Liou, C.-D. (2013). Medium-term multi-plant capacity planning problems considering auxiliary tools for the semiconductor foundry, International Journal of Advanced Manufacturing Technology, Vol. 64, No. 9-12, 1213-1230, doi:10.1007/s00170-0124080-9

[16] Tomlin, B.; Wang, Y. (2005). On the value of mix flexibility and dual sourcing in unreliable newsvendor network, Manufacturing \& Service Operations Management, Vol. 7, No. 1, 37-57, doi: $10.1287 / \mathrm{msom} .1040 .0063$

[17] Claro, J.; de Sousa, J. P. (2012). A multiobjective metaheuristic for a mean-risk multistage capacity investment problem with process flexibility, Computers \& Operations Research, Vol. 39, No. 4, 838-849, doi:10.1016/j.cor.2010.08.015

[18] Malhotra, M. K.; Mackelprang, A. W. (2012). Are internal manufacturing and external supply chain flexibilities complementary capabilities?, Journal of Operations Management, Vol. 30, No. 3, 180-200, doi:10.1016/j.jom.2012.01.004

[19] Hendricks, K. B.; Singhal, V. R. (2005). An empirical analysis of the effect of supply chain disruptions on long-run stock price performance and equity risk of the firm, Production and Operations Management, Vol. 14, No. 1, 35-52, doi:10.1111/j.1937-5956.2005.tb00008.x

[20] Yang, L.; Wang, Y.; Ma, J.; Ng, C. T.; Cheng, T. C. E. (2014). Technology investment under flexible capacity strategy with demand uncertainty, International Journal of Production Economics, Vol. 154, 190-197, doi:10.1016/j.ijpe.2014.04.008

[21] Gino, F.; Pisano, G. (2008). Toward a theory of behavioral operations, Manufacturing \& Service Operations Management, Vol. 10, No. 4, 676-691, doi:10.1287/msom.1070.0205

[22] Schweitzer, M. E.; Cachon, G. P. (2000). Decision bias in the newsvendor problem with a known demand distribution: experimental evidence, Management Science, Vol. 46, No. 3, 404-420, doi:10.1287/mnsc.46.3.404.12070

[23] Camerer, C. F. (2000). Prospect theory in the wild: evidence from the field, Ch. 15, Kahneman, D.; Tversky, A. (Eds.), Choices, values and frames, Cambridge University Press, Cambridge, 288-300

[24] Cachon, G. P.; Terwiesch, C. (2009). Matching Supply with Demand, McGraw-Hill, Singapore

[25] Corbett, C. J.; Rajaram, K. (2006). A generalization of the inventory pooling effect to nonnormal dependent demand, Manufacturing \& Service Operations Management, Vol. 8, No. 4, 351-358, doi:10.1287/msom.1060.0117

[26] Berger, P. D.; Gerstenfeld, A.; Zeng, A. Z. (2004). How many suppliers are best? A decisionanalysis approach, Omega, Vol. 32, No. 1, 9-15, doi:10.1016/j.omega.2003.09.001

[27] De Giorgi, E. G.; Post, T. (2011). Loss aversion with a state-dependent reference point, Management Science, Vol. 57, No. 6, 1094-1110, doi:10.1287/mnsc.1110.1338 